\title{
A simple technique for growing large, optically "perfect" ice crystals
}

\author{
Charles A. KNight \\ National Center for Atmospheric Research, P.O. Box 3000, Boulder, Colorado 80307, U.S.A.
}

\begin{abstract}
Large, single ice crystals containing no air bubbles and free of both small-angle grain boundaries and visible stress birefringence can be grown using a very simple grow th chamber within a temperature-controlled, outer enclosure. The method relies upon the spontaneous formation of an ice crystal with its $c$ axis accurately normal to a free, slightly supercooled, water surface.
\end{abstract}

\section{INTRODUCTION}

Ice single crystals are useful for many laboratory experiments. Decades ago, a common source of such crystals was natural glacier ice, often from Mendenhall Glacier near Juneau, Alaska (e.g. Nakaya, 1956). Large ice crystals have also been grown by standard methods (e.g. Higashi, 1974; Roos, 1975) and others (Turner and others, 1987). This note describes a method that has been in use at the National Center for Atmospheric Research for several years, that is somewhat simpler than most others and produces large crystals that are optically perfect according to naked-eye inspection using polarized light.

\section{THE BASIC METHOD}

All versions of this technique have employed a commercial, constant-temperature incubator set between $-0.5^{\circ}$ and $-1.0^{\circ} \mathrm{C}$ and constant within a few tenths of a degree. The crystal-growth chamber within the incubator is a large, heavily insulated, flat-bottomed, polycarbonate tub. A $250 \mathrm{ml}$ plastic container with a hole in the bottom was put into the tub, filled with air and enough stainlesssteel weights to hold it at the bottom, under water. The tub is filled with water and placed in the incubator.

The purpose of the plastic container in the bottom of the growth chamber is pressure relief. During freezing, water is forced into the container, compressing the air so that the ice disc is not cracked by the expansion as it grows. This also discourages air-bubble formation by preventing the sudden pressure decreases that accompany cracking. The relatively slight rise in pressure as the air in the bottle is compressed probably helps too, since it increases the solubility of air in the water. The water used was processed through a high-quality deionizing system, but was not degassed.

Using a tub $25 \mathrm{~cm}$ deep and $21 \mathrm{~cm}$ in diameter, with about $4 \mathrm{~cm}$ of foam insulation and water at room temperature, ice starts forming after about $1 \mathrm{~d}$ and thereafter thickens at roughly $1 \mathrm{~cm} \mathrm{~d}^{-1}$. Much of the ice slab that forms can be a crystal with its $c$ axis accurately vertical and with no optical evidence of imperfection. The slab often is largely free of air bubbles until it reaches a thickness of 5 or $6 \mathrm{~cm}$. Once in about ten tries an ice disc 5 or $6 \mathrm{~cm}$ thick would be a single, "perfect" crystal $21 \mathrm{~cm}$ in diameter. One in two or three tries would furnish a crystal with cross-sectional area of $100 \mathrm{~cm}^{2}$ or more.

No direct control of crystal initiation or of the nuclei for air-bubble formation at the ice/water interface proved necessary for the production of good crystals. The large crystals are stored at $-15^{\circ}$ to $-20^{\circ} \mathrm{C}$, but they cannot be cooled to that temperature very fast without running the risk of cracking from the thermal stress.

\section{THE PERFECTION OF THE GRYSTALS}

No studies have been undertaken to establish the dislocation or impurity content of the crystals grown with this technique, since neither was important for our purpose, which was to produce many oriented "seeds" for growth experiments. However, the optical perfection is remarkable. It is also remarkable that such perfection is present only when the crystal $c$ axis is accurately normal to the water surface. (It would be difficult and probably pointless to quantify this.) Figure 1 shows the central, colorless portions of optic-axis interference figures from ice slabs $2.5 \mathrm{~cm}$ thick. The close-up camera was focused at the middles of the slabs, and all panels were photographed and printed with the same exposures and contrast, so that the differences in overall appearance between panels owe little to the photography. Figure la shows one of the perfect crystals. The interference figure appears flawless to the naked eye. Figure $1 \mathrm{~b}$ is from a single crystal that grew with its $c$ axis about $3^{\circ}$ from vertical, and shows marked optical inhomogeneity. Figure lc is included to show the striking (and puzzling) stress birefringence that always accompanies 


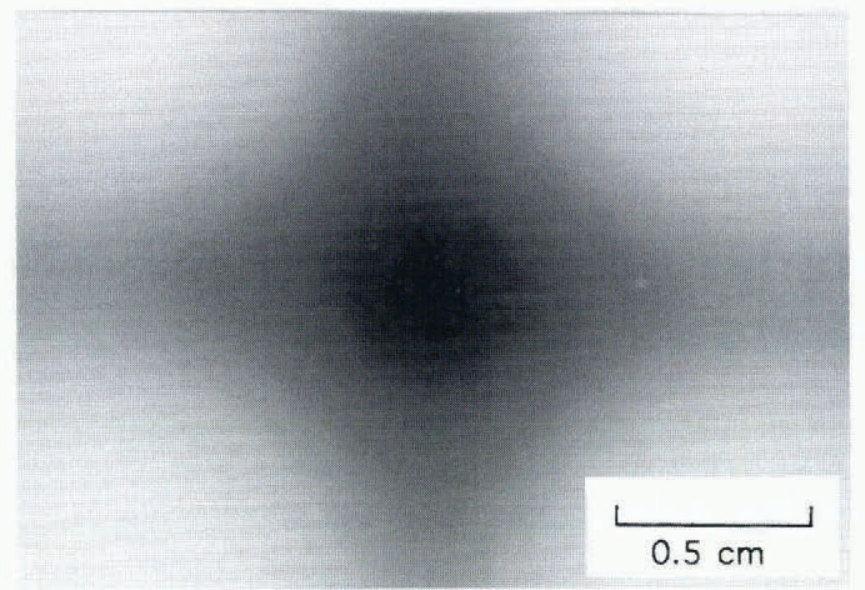

a

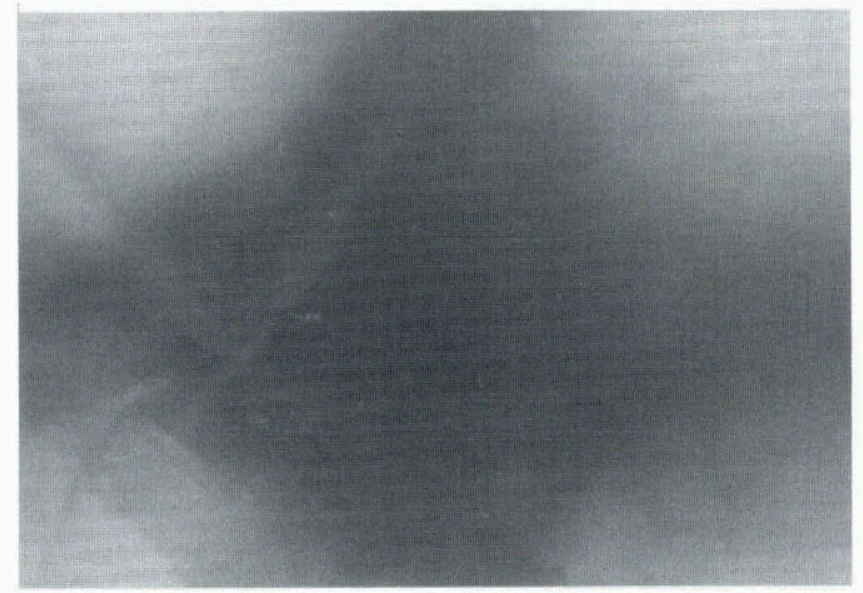

b

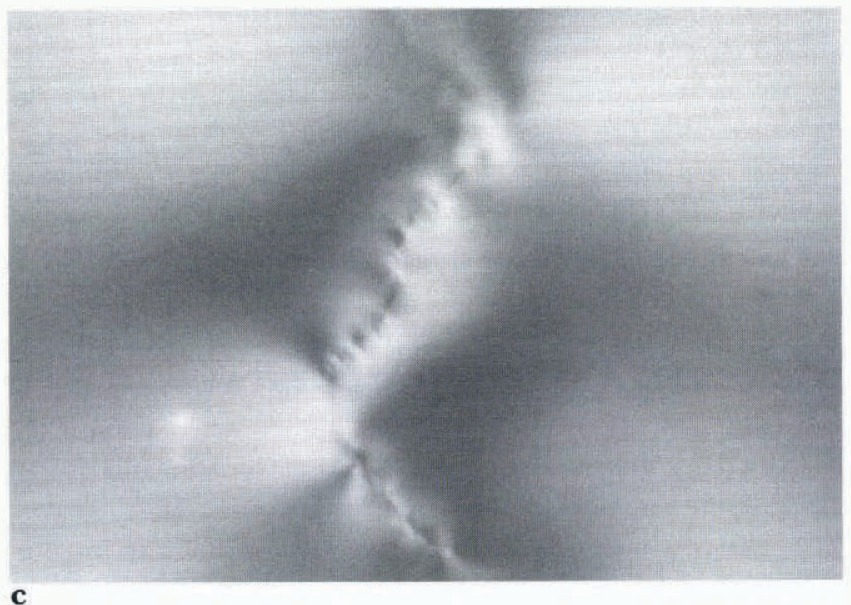

Fig. 1. Optic-axis interference figures from slabs $2.5 \mathrm{~cm}$ thick, of (a) an optically "perfect" crystal, (b) an imperfect crystal that grew with ils optic axis about $3^{\circ}$ from the vertical, and (c) a bicrystal, showing stress birefringence from the grain boundary between two "perfect" crystals. The scale represents the plane in focus, though it would not be very different at either the top or the bottom of the slab. A $55 \mathrm{~mm}$ lens was used with $1: 1$ magnification onto the film

grain boundaries between crystals with their $c$ axes parallel, and is not seen accompanying other grain boundaries. The two adjoining crystals were both optically perfect in this case, except close to their grain boundary, which itself is parallel to the $c$ axis. (This stress birefringence is also seen in sections that are thin, but is not evident in sections cut parallel to the $c$ axis, either normal or parallel to the grain boundary. Thus it presumably represents strain that does not involve local tilting of the $c$ axes. The stress birefringence shows little or no decrease upon annealing for weeks at about $-10^{\circ} \mathrm{C}$.)

Crystals that grow with their $c$ axes at high angles to the water surface (more than perhaps 15 or $20^{\circ}$, but this figure is poorly established) also show no stress birefringence, but the big ones commonly contain small-angle grain boundaries.

The optically "perfect" crystals are excellent for growing melt figures (Tyndall figures) by exposing the ice at $0^{\circ} \mathrm{C}$ to infrared radiation. Few Tyndall figures nucleate, so they often grow to over a centimeter in diameter. Many smaller Tyndall figures form under the same conditions in any crystal that grew with its $c$ axis other than vertical.

\section{WHY DOES THIS METHOD WORK?}

After the chamber filled with water is placed within the constant-temperature enclosure, the water cools from its upper surface, and mixes by convection until its temperature reaches $4^{\circ} \mathrm{C}$. Then the cooler water no longer sinks, the surface layer supercools and freezing can commence. Nucleation of ice is a very rare event at $-0.5^{\circ}$ to $-1.0^{\circ} \mathrm{C}$, and we suspect that the start of freezing rarely if ever is a nucleation process, but is caused by one or more tiny frost crystals dislodged from the refrigeration unit impacting the water surface. If that piece of frost is a single crystal, it grows fastest in the basal direction, and buoyancy forces orient it with its $c$ axis vertical. Then it grows laterally in all directions to cover the surface.

This reorientation is not achieved if the ice that falls into the water is a polycrystal. Also, the growth sometimes appears to start at the wall, in which case also the crystal cannot reorient. However, even if one or a small number of crystals start with non-vertical $c$ axes, room may still remain for desirably oriented crystals to form in the remaining liquid surface before it becomes covered. This also is because the growth rate of ice at low supercooling is very much faster normal than parallel to $c$. Any orientation except with the $c$ axis vertical forms blades that extend across the surface and into the water but thicken very slowly. Open water at the surface then lasts $1 \mathrm{~h}$ or more, leaving opportunity for a new crystal with its $c$ axis vertical to cover the remaining area of open water.

Since the orientation of the starting ice is easily determined by inspection, it pays to look at the ice during the first freezing, and start over if most of the surface is covered by crystals with the wrong orientation.

Why must the initial crystal have its $c$ axis accurately vertical for this method to produce the crystals with excellent optical quality? The answer to this question very likely relates to the beginning crystal morphology. A crystal with its $c$ axis even a little off vertical grows as a basal plate extending down into the water at an angle. When that angle is more than a few degrees, the plate is observed to extend as much as $1 \mathrm{~cm}$ below the water surface before coverage with ice is complete. This also is a common observation in the freezing of open puddles, 
perhaps first noted by Hallett (1960). When the basal blades extend into the water at an angle, buoyancy forces may be sufficient to deform the ice enough to generate small-angle grain boundaries and other imperfections. Also, soluble impurities may become trapped in the ice between the blades. Then the expansion from partial freezing of these included pockets of solution can cause small-angle grain boundaries (Knight, 1962).

\section{IMPROVEMENTS}

Several improvements of this method have been attempted. In one, an outlet at the chamber bottom with a tube to discharge water into a container at a higher level within the constant-temeprature enclosure keeps the water pressure constant as the ice disc thickens. This works well, there is little or no problem of the tube freezing up if the discharge end of the tube is well shielded, and having the tube into the bottom of the growth chamber also greatly simplifies removal of the ice disc. However, air-bubble formation appears to be more of a problem without the pressure increase. This can be solved by providing very gentle stirring, using a magnetic stirring bar controlled at $100 \mathrm{rpm}$, started when the ice thickness reaches $0.5-1 \mathrm{~cm}$. Stirring too vigorously or too early melts the ice. Another improvement is to provide very gentle heating around the container at the level of the water surface to discourage initial crystal formation at the walls. This heating is turned off as soon as the surface is ice-covered.

However, the basic method has been more than adequate to supply oriented, single-crystal seeds for other crystal-growth experiments. The $c$-axis orientation is known, and $a$-axis orientations can be determined by inspection of frost crystals grown on the basal surface. Oriented slabs can then be cut on a band-saw, and the seeds cut from these oriented slabs. Occasional air bubbles in the ice were not a problem in making seeds about $1.5 \mathrm{~cm}$ on a side. The more elaborate system, with stirring to prevent high supersaturation with air at the water/ice interface, is recommended if single crystals considerably larger than that are required to be completely free of air bubbles.

\section{ACKNOWLEDGEMENT}

The National Center for Atmospheric Research is sponsored by the U.S. National Science Foundation.

\section{REFERENCES}

Hallett, J. 1960. Crystal growth and the formation of spikes in the surface of supercooled water. J. Glaciol., 3 28), 698-704.

Higashi, A. 1974. Growth and perfection of ice crystals. 7. Cryst. Growth, 24-25, 102-107.

Knight, C. A. 1962. Migration of liquid inclusions parallel to the $c$-axis in ice single crystals: an origin for some striations. Can. J. Phys., 40 , $1681-1685$.

Nakaya, U. 1956. Properties of single crystals of ice, revealed by internal melting. SIPRE Res. Pap. 13.

Roos, D.v.d.S. 1975. Rapid production of single crystals of ice. $\mathcal{J}$. Glaciol., 14 (71), 325-328.

Turner, G.J., C. D. Stow and R. Keatinge. 1987. The manufacture of large samples of monocrystaline ice under microcomputer control. $\mathcal{J}$. Cryst. Growth, 80 (2), 463-464. 